\title{
Uniformly accelerated finite-time detectors
}

\author{
A. Higuchi \\ The University of Chicago, The Enrico Fermi Institute, 5640 S. Ellis Avenue, Chicago, Illinois 60637-1433
}

G. E. A. Matsas

Universidade de São Paulo, Instituto de Física, Cx.P. 20516, 01498-970-São Paulo, São Paulo, Brazil

and Instituto de Física Teórica-Universidade Estadual Paulista, Rua Pamplona 145, 01405-900-São Paulo, São Paulo, Brazil

C. B. Peres

Instituto de Física Teórica-Universidade Estadual Paulista, Rua Pamplona 145, 01405-900-São Paulo, São Paulo, Brazil

(Received 17 June 1993)

\begin{abstract}
The behavior of uniformly accelerated detectors in the Minkowski and Rindler vacua is analyzed when the detector is coupled to a scalar field during a finite amount of time $T$. We point out that the logarithmic ultraviolet divergences reported in the literature are due to the instantaneous switching of the detector. We explicitly show this by considering a detector switched on and off continuously. The usual Planckian spectrum for the excitation probability is recovered in the limit $T \rightarrow+\infty$.
\end{abstract}

PACS number(s): 04.60. $+\mathrm{n}, 03.70 .+\mathrm{k}$

The development of quantum-field theory in curved space-time has raised a number of questions related to the particle concept and its frame dependence. As a consequence, the behavior of detectors moving in various ways has been a continuous source of renewed investigation (see [1] for a comprehensive discussion). It is known that a uniformly accelerated detector in the Minkowski vacuum feels a thermal bath characterized by a temperature proportional to its proper acceleration. This thermal bath is often called the Fulling-Davies-Unruh (FDU) thermal bath $[2,3]$, and it is composed of Rindler particles. Thus, a uniformly accelerated detector prepared in the ground state has a finite probability of being excited to a higher level in the Minkowski vacuum. This transition is followed by the emission of a Minkowski particle as described by inertial observers, and by the absorption of a Rindler particle from the FDU thermal bath as described by uniformly accelerated observers [4]. The introduction of the FDU thermal bath is also crucial in analyzing other quantum phenomena such as the bremsstrahlung effect in the accelerated frame [5].

The FDU thermal bath is usually analyzed with an idealized detector which is turned on forever. It would be more desirable to consider finite-time detectors to see what would actually happen to realistic detectors. (By finite-time detectors we mean those detectors which interact with the field during a finite amount of time $T$.) Recently the excitation rate associated with uniformly accelerated finite-time detectors interacting with the Minkowski vacuum has been analyzed in the inertial frame by Svaiter and Svaiter [6]. They find that the excitation rate is divergent. In order to solve this problem they use a "renormalization" procedure. However, as they point out themselves, their procedure does not guarantee that the final expression for the excitation rate is positive definite.

In this paper we show that no divergence appears in the excitation rate for finite-time detectors provided that they are turned on and off continuously. (Actually, this is a more realistic procedure for modeling physical detectors.) Moreover, no "renormalization" procedure is used here, and the excitation probability obtained is positive definite. We also obtain an expression for the excitation rate in the limit where the detector is kept switched on for a long time. The usual Planckian spectrum is recovered when we keep the detector permanently turned on $(T \rightarrow+\infty)$. The analysis is carried out in the uniformly accelerated frame, where the calculations are simpler. Finally, we show that our conclusions do not depend sensitively on the way the detector is switched on and off. We will carry out our analysis with Unruh-DeWitt detectors. The metric signature adopted is $(+---)$, and natural units $\left(\hbar=c=k_{B}=1\right)$ are used.

In order to analyze the behavior of a uniformly accelerated finite-time detector in its rest frame, we will first quantize the massless scalar field in the Rindler wedge, i.e., the portion $z>|t|$ of the Minkowski space-time. We use Rindler coordinates $(\tau, x, y, \xi)$ to cover the Rindler wedge, which are related to the usual Minkowski coordinates $(t, x, y, z)$ by (see, e.g., [7])

$$
t=\frac{e^{a \xi}}{a} \sinh a \tau, z=\frac{e^{a \xi}}{a} \cosh a \tau .
$$

A uniformly accelerated observer with its motion constrained to the $t z$ plane, and with constant proper acceleration $a$ has a hyperbolic world line in Minkowski space-time. In Rindler coordinates this world line is simply $x=y=\xi=0$. Using (1), the Minkowski line element restricted to the Rindler wedge is written as

$$
d s^{2}=e^{2 a \xi}\left(d \tau^{2}-d \xi^{2}\right)-d x^{2}-d y^{2} .
$$

The timelike Killing field with respect to which we quantize the fields in the Rindler wedge is the boost Killing field $\partial_{\tau}$. The boundary $z=|t|(\xi=-\infty)$ is a Killing horizon with respect to $\partial_{\tau}$.

The field quantization in the Rindler wedge proceeds in the standard way (see, e.g., Ref. [8]). Consider a mass- 
less scalar field $\phi(x)$ satisfying the usual Klein-Gordon equation

$$
\square \phi=0 .
$$

A complete set of normal modes satisfying (3) in the Rindler wedge (and asymptotically well behaved for $\xi \rightarrow$ $+\infty)$ has the form

$$
\begin{aligned}
u_{\omega \mathbf{k}_{\perp}}\left(x^{\mu}\right)= & {\left[\frac{\sinh (\pi \omega / a)}{4 \pi^{4} a}\right]^{1 / 2} } \\
& \times K_{i \omega / a}\left(\frac{k_{\perp}}{a} e^{a \xi}\right) e^{i \mathbf{k}_{\perp} \cdot \mathbf{x}_{\perp}-i \omega \tau} .
\end{aligned}
$$

Each Rindler mode is labeled by its frequency $\omega$, and transverse momentum $\mathbf{k}_{\perp} \equiv\left(k_{x}, k_{y}\right) . K_{i \nu}(x)$ is the MacDonald function, $k_{\perp} \equiv \sqrt{k_{x}^{2}+k_{y}^{2}}$, and $\mathbf{x}_{\perp} \equiv(x, y)$. Notice that the modes given by (4) with $\omega>0$ are positive frequency modes with respect to $\partial_{\tau}$. The scalar field is Fourier decomposed in terms of these modes as

$$
\hat{\phi}\left(x^{\nu}\right)=\int d^{2} \mathbf{k}_{\perp} \int_{0}^{+\infty} d \omega\left\{\hat{a}_{\omega \mathbf{k}_{\perp}}^{R} u_{\omega \mathbf{k}_{\perp}}\left(x^{\nu}\right)+\text { H.c. }\right\}
$$

where $\hat{a}_{\omega \mathbf{k}_{\perp}}^{R}$ and $\hat{a}_{\omega \mathbf{k}_{\perp}}^{R \dagger}$ are the annihilation and creation operators, respectively, of Rindler modes. Modes (4) are normalized with respect to the Klein-Gordon inner product:

$$
\left(u_{\omega \mathbf{k}_{\perp}}, u_{\omega^{\prime} \mathbf{k}_{\perp}^{\prime}}\right)=\delta\left(\omega-\omega^{\prime}\right) \delta\left(\mathbf{k}_{\perp}-\mathbf{k}_{\perp}^{\prime}\right)
$$

where

$$
\left(u_{\omega \mathbf{k}_{\perp}}, u_{\omega^{\prime} \mathbf{k}_{\perp}^{\prime}}\right) \equiv i \int_{\Sigma} d \Sigma^{\mu} \sqrt{-g_{\Sigma}} u_{\omega \mathbf{k}_{\perp}}^{*} \stackrel{\leftrightarrow}{\partial_{\mu}} u_{\omega^{\prime} \mathbf{k}_{\perp}^{\prime}}
$$

Here $d \Sigma^{\mu}=n^{\mu} d \Sigma$, where $n^{\mu}$ is the future directed unit normal to a Cauchy surface $\Sigma$. (A convenient Cauchy surface in our case is $\tau=$ const.) With this normalization, and using the usual canonical commutation relations for the field, we obtain

$$
\left[\hat{a}_{\omega \mathbf{k}_{\perp}}^{R}, \hat{a}_{\omega^{\prime} \mathbf{k}_{\perp}^{\prime}}^{R \dagger}\right]=\delta\left(\omega-\omega^{\prime}\right) \delta\left(\mathbf{k}_{\perp}-\mathbf{k}_{\perp}^{\prime}\right) .
$$

All the other commutators vanish. The Rindler vacuum $|0\rangle_{R}$, i.e., the no particle state with respect to uniformly accelerated observers, is defined by $\hat{a}_{\omega \mathbf{k}}^{R}|0\rangle_{R}=0$.

Now, consider a detector following a world line $x^{\mu}(\tau)$, where $\tau$ is its proper time. The Unruh-DeWitt detector is modeled by a two-level monopole described by the operator $\hat{m}(\tau)$. In addition, it is coupled to the scalar field through the interaction action

$$
\mathcal{S}_{I}=\int_{-\infty}^{+\infty} d \tau c(\tau) \hat{m}(\tau) \hat{\phi}\left[x^{\mu}(\tau)\right]
$$

where the function

$$
c(\tau) \equiv c_{0}\left\{\begin{array}{cc}
e^{\alpha(\tau+T)}, & \tau<-T \\
1, & -T \leq \tau \leq T \\
e^{-\alpha(\tau-T)}, & \tau>T
\end{array}\right.
$$

has been inserted to switch off the detector continuously as $\tau \rightarrow \pm \infty$. Here $c_{0}$ is a small coupling constant, and $\alpha \in \mathbb{R}_{+}$. The monopole operator evolves in time as

$$
\hat{m}(\tau)=e^{i H_{0} \tau} \hat{m}(0) e^{-i H_{0} \tau},
$$

where $H_{0}$ is the free Hamiltonian associated with the detector, i.e., $H_{0}|E\rangle=E|E\rangle$ for any detector's eigenstate $|E\rangle$.

Before we analyze the behavior of the detector in the Minkowski vacuum, we consider the detector's excitation probability with simultaneous emission of a Rindler particle in the Rindler vacuum. The amplitude associated with this process in first order of perturbation is

$$
\mathcal{A}_{\mathrm{em}}^{R}={ }_{R}\left\langle\omega \mathbf{k}_{\perp}\left|\otimes\left\langle E\left|\mathcal{S}_{I}\right| E_{0}\right\rangle \otimes\right| 0\right\rangle_{R} .
$$

The differential probability associated with this amplitude is

$$
d W_{\mathrm{em}}^{R}=\left|\mathcal{A}_{\mathrm{em}}^{R}\right|^{2} d^{2} \mathbf{k}_{\perp} d \omega .
$$

As mentioned in the beginning, however, an observer with constant proper acceleration $a$, in the Minkowski vacuum, detects in its rest frame a thermal bath characterized by a temperature $T=a / 2 \pi$. We emphasize here that the Minkowski vacuum is indistinguishable from the thermal bath built on the Rindler vacuum as long as the detector stays in the Rindler wedge. This fact follows immediately from the work of Unruh [3] who explicitly expressed the Minkowski vacuum as a linear combination of products of the left and right Rindler states. For this reason, the detector's excitation probability with simultaneous emission of a Rindler particle into the Minkowski vacuum is given by (12) combined with the proper thermal factor

$$
\mathcal{P}_{\mathrm{em}}^{\mathrm{ex}}=\int d W_{\mathrm{em}}^{R}\left(1+\frac{1}{e^{2 \pi \omega / a}-1}\right) .
$$

The two terms in the parentheses are associated with the spontaneous and induced emission, respectively.

Similarly one can calculate the detector's excitation probability with simultaneous absorption of a Rindler particle from the FDU thermal bath associated with the Minkowski vacuum

$$
\mathcal{P}_{\mathrm{abs}}^{\mathrm{ex}}=\int d W_{\mathrm{abs}}^{R} \frac{1}{e^{2 \pi \omega / a}-1}
$$

where

$$
d W_{\mathrm{abs}}^{R} \equiv\left|\mathcal{A}_{\mathrm{abs}}^{R}\right|^{2} d^{2} \mathbf{k}_{\perp} d \omega
$$

and

$$
\mathcal{A}_{\mathrm{abs}}^{R}={ }_{R}\left\langle 0\left|\otimes\left\langle E\left|\mathcal{S}_{I}\right| E_{0}\right\rangle \otimes\right| \omega \mathbf{k}_{\perp}\right\rangle_{R}
$$

Now we recall that each absorption or emission of a Rindler particle (associated with the detector's excitation) as described by uniformly accelerated observers corresponds uniquely to the emission of a Minkowski particle as described by inertial observers [4]. Since in first order of perturbation there is no interference, 
the total probability for this detector to be excited in the Minkowski vacuum (with simultaneous emission of a Minkowski particle) is (see Refs. [5,9] for the case in which the detector is replaced by a classical charge)

$$
\mathcal{P}^{M}=\mathcal{P}_{\text {em }}^{\text {ex }}+\mathcal{P}_{\text {abs }}^{\text {ex }} \text {. }
$$

Before evaluating this expression, we need to obtain the amplitudes (11) and (16). Using (5), and (8)-(10) we get

$$
\begin{aligned}
\mathcal{A}_{\substack{\mathrm{abs} \\
\mathrm{abs}}}^{R}= & c_{0}\left\langle E|\hat{m}(0)| E_{0}\right\rangle\left[\frac{\sinh (\pi \omega / a)}{4 \pi^{4} a}\right]^{1 / 2} K_{i \omega / a}\left(\frac{k_{\perp}}{a}\right) \\
\times & {\left[\frac{2 \sin (\omega \pm \Delta E) T}{\omega \pm \Delta E}+\frac{2 \alpha \cos (\omega \pm \Delta E) T}{\alpha^{2}+(\omega \pm \Delta E)^{2}}\right.} \\
& \left.-\frac{2(\omega \pm \Delta E) \sin (\omega \pm \Delta E) T}{\alpha^{2}+(\omega \pm \Delta E)^{2}}\right]
\end{aligned}
$$

where $\Delta E=E-E_{0}>0$. Next, we calculate (17) by using (13) and (14). After integrating in the transverse momentum [10], we obtain

$$
\mathcal{P}^{M}=\frac{c_{0}^{2}}{4 \pi^{2}}\left|\left\langle E|\hat{m}(0)| E_{0}\right\rangle\right|^{2}\left[I_{\mathrm{sp}}+I_{\mathrm{in}}+I_{\mathrm{abs}}\right],
$$

where

$$
\begin{gathered}
I_{\mathrm{sp}}=\int_{0}^{+\infty} d \omega \omega B(\omega), \\
I_{\mathrm{in}}=\int_{0}^{+\infty} d \omega g(\omega) B(\omega), \\
I_{\mathrm{abs}}=\int_{0}^{+\infty} d \omega g(\omega) B(-\omega) .
\end{gathered}
$$

Here we have defined

$$
\begin{aligned}
B(\omega) \equiv & \frac{4 \sin ^{2}(\omega+\Delta E) T}{(\omega+\Delta E)^{2}}-\frac{4 \sin ^{2}(\omega+\Delta E) T}{\alpha^{2}+(\omega+\Delta E)^{2}} \\
& +\frac{4 \alpha^{2} \cos 2(\omega+\Delta E) T}{\left[\alpha^{2}+(\omega+\Delta E)^{2}\right]^{2}} \\
& +\frac{4 \alpha^{3} \sin 2(\omega+\Delta E) T}{(\omega+\Delta E)\left[\alpha^{2}+(\omega+\Delta E)^{2}\right]^{2}}
\end{aligned}
$$

and

$$
g(\omega) \equiv \frac{\omega}{e^{2 \pi \omega / a}-1}
$$

Let us first show that $\mathcal{P}^{M}$ in (19) is finite. It is easy to notice from (20)-(22) that the integrand of (19) does not diverge at any isolated point. Also, the leading term of the asymptotic expansion $(\omega>>\Delta E, \alpha, a)$ of this integrand is $4 \alpha^{2} \omega^{-3} \cos ^{2} \omega T$. Thus, (19) is clearly finite. Let us now suppose we instantaneously switch on and after a while we instantaneously switch off the detector, in such a way that it only interacts with the field during the interval $-T<\tau<T$. It corresponds to the limit $\alpha \rightarrow+\infty$ [see Eq. (9)]. In this regime the integrand of (19) behaves asymptotically like $4 \omega^{-1} \sin ^{2} \omega T$ which gives rise to a logarithmic ultraviolet divergence.

In order to analyze (19) more carefully for large $T$ (i.e., $\left.T>>a^{-1}, \alpha^{-1}, \Delta E^{-1}\right)$, we note in this regime the useful relations

$$
\begin{gathered}
\int_{a}^{b} d \omega f(\omega) \sin 2(\omega+\Delta E) T \stackrel{\text { large } T}{\approx} 0, \\
\int_{a}^{b} d \omega f(\omega) \sin ^{2}(\omega+\Delta E) T \stackrel{\text { large } T}{\approx} \frac{1}{2} \int_{a}^{b} d \omega f(\omega),
\end{gathered}
$$

where $f(\omega)$ is any finite function satisfying $\int_{a}^{b}|f(\omega)| d \omega<$ $+\infty$. Notice that the relations (25) and (26) are exact for $T \rightarrow+\infty$, and that they are also valid with "sin" replaced by "cos."

It is easy to evaluate (20) using (25) and (26), since the last two terms of (23) may be neglected:

$$
I_{\mathrm{sp}} \stackrel{\text { large } T}{\approx} \ln \frac{\alpha^{2}+\Delta E^{2}}{\Delta E^{2}}-2+\frac{\Delta E}{\alpha}\left[\pi-2 \arctan \frac{\Delta E}{\alpha}\right] .
$$

Similarly we obtain

$$
I_{\text {in }} \stackrel{\text { large } T}{\approx} 2 \int_{0}^{+\infty} d \omega\left[\frac{g(\omega)}{(\omega+\Delta E)^{2}}-\frac{g(\omega)}{\alpha^{2}+(\omega+\Delta E)^{2}}\right] .
$$

It remains for us to evaluate (22). For this purpose, it has to be cast into a suitable form so that the conditions for the validity of (25) and (26) are satisfied. After some manipulation we obtain

$$
\begin{aligned}
I_{\mathrm{abs}}= & \int_{-\infty}^{+\infty} d \omega \frac{4 g(\Delta E)}{(\omega-\Delta E)^{2}} \sin ^{2}(\omega-\Delta E) T-\int_{-\infty}^{0} d \omega \frac{4 g(\Delta E)}{(\omega-\Delta E)^{2}} \sin ^{2}(\omega-\Delta E) T \\
& +\int_{0}^{+\infty} d \omega \frac{4\left[g(\omega)-g(\Delta E)-\theta(2 \Delta E-\omega)(\omega-\Delta E) g^{\prime}(\Delta E)\right]}{(\omega-\Delta E)^{2}} \sin ^{2}(\omega-\Delta E) T \\
& -\int_{0}^{+\infty} d \omega \frac{4 g(\omega)}{\alpha^{2}+(\omega-\Delta E)^{2}} \sin ^{2}(\omega-\Delta E) T \\
& +\int_{0}^{+\infty} d \omega \frac{4 \alpha^{2} g(\omega)}{\left[\alpha^{2}+(\omega-\Delta E)^{2}\right]^{2}} \cos 2(\omega-\Delta E) T \\
& +\int_{0}^{+\infty} d \omega \frac{4 \alpha^{3}}{\omega-\Delta E}\left[\frac{g(\omega)}{\left[\alpha^{2}+(\omega-\Delta E)^{2}\right]^{2}}-\frac{g(\Delta E) \theta(2 \Delta E-\omega)}{\alpha^{4}}\right] \sin 2(\omega-\Delta E) T \\
& +\int_{0}^{+\infty} d \omega \frac{4 \alpha^{3}}{\omega-\Delta E} \frac{g(\Delta E) \theta(2 \Delta E-\omega)}{\alpha^{4}} \sin 2(\omega-\Delta E) T,
\end{aligned}
$$


where the prime denotes the derivative with respect to the argument. The last term of the third integral does not contribute, but it was introduced because it enables us to use (26). Now, using (25) and (26) to estimate all the integrals but the first and last ones, which can be evaluated directly [10], we obtain

$$
I_{\mathrm{abs}} \stackrel{\text { large }}{\approx} 4 g(\Delta E)\left[\pi T-\frac{1}{2 \Delta E}\right]-2 \int_{0}^{+\infty} d \omega \frac{g(\omega)}{\alpha^{2}+(\omega-\Delta E)^{2}}+2 \mathrm{P} \int_{0}^{+\infty} d \omega \frac{g(\omega)-g(\Delta E)}{(\omega-\Delta E)^{2}}+\frac{4 g(\Delta E) \pi}{\alpha}
$$

where $\mathrm{P}$ indicates the principal value.

Substituting (27), (28), and (30) in (19), we obtain

$$
\begin{aligned}
\mathcal{P}^{M} \stackrel{\text { large } T}{\approx} & \frac{c_{0}^{2}}{2 \pi}\left|\left\langle E|\hat{m}(0)| E_{0}\right\rangle\right|^{2} \frac{\Delta E}{e^{2 \pi \Delta E / a}-1} T^{\text {tot }}+\frac{c_{0}^{2}}{4 \pi^{2}}\left|\left\langle E|\hat{m}(0)| E_{0}\right\rangle\right|^{2} \\
& \times\left\{\ln \frac{\alpha^{2}+\Delta E^{2}}{\Delta E^{2}}-2+\frac{\pi \Delta E}{\alpha}-\frac{2 \Delta E}{\alpha} \arctan \frac{\Delta E}{\alpha}-\frac{2 g(\Delta E)}{\Delta E}+\frac{4 \pi g(\Delta E)}{\alpha}\right. \\
& \left.-2 \mathrm{P} \int_{-\infty}^{+\infty} d \omega \frac{g(|\omega|)}{\alpha^{2}+(\omega-\Delta E)^{2}}+2 \mathrm{P} \int_{-\infty}^{+\infty} d \omega \frac{g(|\omega|)-g(\Delta E)}{(\omega-\Delta E)^{2}}\right\},
\end{aligned}
$$

where $T^{\text {tot }}=2 T$. This expression can be simplified further in the approximation $\alpha>>\Delta E, a$ :

$$
\begin{aligned}
\mathcal{P}^{M} \underset{\approx}{\operatorname{large} T, \alpha} & \frac{c_{0}^{2}}{2 \pi}\left|\left\langle E|\hat{m}(0)| E_{0}\right\rangle\right|^{2} \frac{\Delta E}{e^{2 \pi \Delta E / a}-1} T^{\text {tot }}+\frac{c_{0}^{2}}{2 \pi^{2}}\left|\left\langle E|\hat{m}(0)| E_{0}\right\rangle\right|^{2} \\
& \times\left\{\ln \frac{\alpha}{\Delta E}-1-\frac{1}{e^{2 \pi \Delta E / a}-1}+\mathrm{P} \int_{-\infty}^{+\infty} d \omega \frac{g(|\omega|)-g(\Delta E)}{(\omega-\Delta E)^{2}}\right\} .
\end{aligned}
$$

This is an approximate expression for the excitation probability in the regime $T>>a^{-1}, \Delta E^{-1}$ and $\alpha>>$ $\Delta E, a$. Notice the logarithmic divergence for $\alpha \rightarrow+\infty$. The same divergence appears in the calculation of the detector's excitation rate in the Rindler vacuum, which is obtained by dropping $I_{\text {in }}$ and $I_{\text {abs }}$ in (19). Finally, if we take the limit $T \rightarrow+\infty$ in (32), the first term dominates, and we recover the Planckian form for the average excitation rate (see [7], p. 53):

$$
\left.\frac{\mathcal{P}^{M}}{T^{\text {tot }}}\right|_{T \rightarrow+\infty}=\frac{c_{0}^{2}}{2 \pi}\left|\left\langle E|\hat{m}(0)| E_{0}\right\rangle\right|^{2} \frac{\Delta E}{e^{2 \pi \Delta E / a}-1}
$$

The good ultraviolet behavior of the detector's total excitation probability $\mathcal{P}^{M}$ does not depend sensitively on the particular choice of the function $c(\tau)$. This is because the ultraviolet behavior of $\mathcal{P}^{M}(\omega)$ [defined by $\mathcal{P}^{M} \equiv \int_{0}^{\infty} d \omega \mathcal{P}^{M}(\omega)$ in (19)] will only depend on the smoothness of the function $c(\tau)$. In general, the behavior of the Fourier transform of a function $f(x) \in C^{n}$ for large $\omega$ is $\tilde{f}(\omega) \sim 1 / \omega^{2+n}$. Hence, assuming $c(\tau) \in C^{n}$ the emission and absorption differential probabilities (12) and (15) will behave asymptotically $(\omega>>\Delta E, \alpha, a)$ as

$\left.\left.d W_{\mathrm{em}}^{R}\right|_{\text {large } \omega} \sim d W_{\mathrm{abs}}^{R}\right|_{\text {large } \omega}$

[1] V.L. Ginzburg and V.P. Frolov, Usp. Fiz. Nauk. 153, 633 (1987) [Sov. Phys. Usp. 30, 1073 (1987)].

[2] S.A. Fulling, Phys. Rev. D 7, 2850 (1973); P.C.W. Davies, J. Phys. A 8, 609 (1975).

[3] W.G. Unruh, Phys. Rev. D 14, 870 (1976).

[4] W.G. Unruh and R.M. Wald, Phys. Rev. D 29, 1047 (1984).

[5] A. Higuchi, G.E.A. Matsas, and D. Sudarsky, Phys. Rev. D 45, R3308 (1992); A. Higuchi, G.E.A. Matsas, and D. Sudarsky, ibid. 46, 3450 (1992).

$$
\sim K_{i \omega / a}^{2}\left(\frac{k_{\perp}}{a}\right) \frac{\sinh (\pi \omega / a)}{\omega^{4+2 n}} d \omega d^{2} \mathbf{k}_{\perp}
$$

Integrating over $\mathbf{k}_{\perp}$ in (34), we find from (13), (14), and (17) that $\left.\mathcal{P}^{M}(\omega)\right|_{\text {large } \omega} \sim \omega^{-3-2 n}$. In conclusion, the detector's total excitation probability will not have a bad ultraviolet behavior provided $c(\tau) \in C^{0}$, i.e., if $c(\tau)$ is at least continuous.

In summary, we have analyzed the excitation probability for a model of a realistic detector switched on and off in a continuous manner, thus obtaining finite results. We have also shown that uniformly accelerated finitetime detectors instantaneously switched on and off lead to ultraviolet logarithmic divergences in the excitation probability.

One of us (C.P.) is indebted to Professor V. A. Lugo for discussions. This work was supported in part by the National Science Foundation under Grant No. PHY 9220644 (A.H.), Fundação de Amparo à Pesquisa do Estado de São Paulo, Conselho Nacional de Desenvolvimento Científico e Tecnológico (G.M.), and Coordenadoria de Aperfeiçoamento de Pessoal de Nível Superior (C.P.).

[6] B.F. Svaiter and N.F. Svaiter, Phys. Rev D 46, 5267 (1992).

[7] N.D. Birrell and P.C.W. Davies, Quantum Field Theory in Curved Space (Cambridge University Press, Cambridge, England, 1982).

[8] L.N. Pringle, Phys. Rev. D 39, 2178 (1989).

[9] A. Higuchi and G.E.A. Matsas, Phys. Rev. D 48, 689 (1993).

[10] I.S. Gradshteyn and I.M. Ryzhik, Table of Integrals, Series and Products (Academic, New York, 1980). 